\title{
Probing dark matter self-interaction in the Sun with IceCube-PINGU
}

\section{Chian-Shu Chen*}

Institute of Physics, Academia Sinica, 128 Sec. 2, Academia Rd., Nankang, Taipei 11529, Taiwan

E-mail: chianshuegmail.com

\section{Guey-Lin Lin}

Institute of Physics, National Chiao Tung University, Hsinchu 30010, Taiwan

E-mail: glin@cc.nctu.edu.tw

\section{Yen-Hsun Lin}

Institute of Physics, National Chiao Tung University, Hsinchu 30010, Taiwan

E-mail: chris.py99g@g2.nctu.edu.tw

\begin{abstract}
The halo dark matter (DM) can be gravitationally captured by the Sun. For self-interacting DM (SIDM), we show that the number of DM trapped inside the Sun remains unsuppressed even if the DM-nucleon cross section is negligible. We consider a SIDM model where U(1) gauge symmetry is introduced to account for the DM self-interaction. Such a model naturally leads to isospin violation for DM-nucleon interaction, although isospin symmetry is still allowed as a special case. We show that the indirect detection of DM-induced neutrinos from the Sun can probe those SIDM parameter ranges not yet reachable by direct detections. Those parameter ranges are either the region with a very small dark matter mass (less than a few $\mathrm{GeV}$ ) or the region opened up due to isospin violations.
\end{abstract}

Flavor Physics CP Violation 2015

May 25-29, 2015

Nagoya, Japan

\footnotetext{
* Speaker.
} 


\section{Introduction}

In this paper, the general framework of dark matter (DM denoting $\chi$ ) capture, annihilation and evaperation inside the Sun via DM-nuclei scattering $\left(\sigma_{\chi p}\right)$ and DM-DM scattering $\left(\sigma_{\chi \chi}\right)$ is presented. We take that both the galactic DM and the nuclei inside the Sun follow the thermal distributions. If DM collides with nuclei in the Sun it can be captured by the Sun when its final velocity is smaller than the escape velocity from the Sun. Alternatively, DM trapped inside the Sun will be kicked out if its final velocity after the scattering with the nuclei is larger than the escape velocity. The inclusion of DM self-interaction also have effects on the capture and evaporation of DM inside the Sun. The existence of self-interacting DM (SIDM) [1] was proposed to solve the cusp/core [2], missing satellites [3], and too-big-to-fail [4] problems those are the inconsistencies between the N-body collisionless DM simulation and astrophysical observations on small scale structures. Constraints on the ratio of SIDM cross section to the DM mass, $0.1<\sigma_{\chi \chi} / m_{\chi}<$ $1.0\left(\mathrm{~cm}^{2} / \mathrm{g}\right)$, were obtained from various observations. We found that the total DM trapped in the Sun can be increased if DM self-interaction is included [5]. This implies a considerable excess cosmic rays come from the annihilation of DM in the direction of the Sun. We show the allowed parameter space of $\sigma_{\chi \chi}$ can be tested by measuring such DM indirect signals. For illustrative purpose, we only consider neutrino flux produced by DM annihilating into leptons such as $\chi \chi \rightarrow$ $\tau^{+} \tau^{-}$and $\chi \chi \rightarrow \nu \bar{v}$. The final-state neutrinos can be detected by the IceCube-PINGU. In the case of suppressed $\sigma_{\chi p}$, we find the total DM number to be accumlated is only slightly less than the scenario of unsuppressed $\sigma_{\chi p}$ [6]. It means that $\sigma_{\chi \chi}$ plays an important role in trapped DM in the Sun. Therefore, we have a significant DM indirect signal even if the direct detection of $\sigma_{\chi p}$ is not very promising. We use this feature to show the complementary probe of SIDM between direct and indirect detections.

\section{Dark matter accumulation in the Sun}

The general DM evolution equation in the Sun is given by

$$
\frac{d N_{\chi}}{d t}=C_{c}+\left(C_{s}-C_{e}\right) N_{\chi}-\left(C_{a}+C_{s e}\right) N_{\chi}^{2}
$$

with $N_{\chi}$ the DM number in the Sun, $C_{c}$ the rate at which DM are captured by the Sun $[7,8]$,

$$
C_{c}^{\mathrm{SI}} \simeq 1.24 \times 10^{24} \mathrm{~s}^{-1}\left(\frac{\rho_{0}}{0.3 \mathrm{GeV} / \mathrm{cm}^{3}}\right)\left(\frac{270 \mathrm{~km} / \mathrm{s}}{\bar{v}}\right)^{3}\left(\frac{\mathrm{GeV}}{m_{\chi}}\right)^{2}\left(\frac{2.6 \sigma_{\mathrm{H}}^{\mathrm{SI}}+0.175 \sigma_{\mathrm{He}}^{\mathrm{SI}}}{10^{-6} \mathrm{pb}}\right)
$$

Here $\sigma_{\mathrm{H}}^{\mathrm{SI}}$ and $\sigma_{\mathrm{He}}^{\mathrm{SI}}$ are SI DM-hydrogen and -helium cross sections respectively. We note that $C_{c}$ can be categorized by the type of interactions between DM particles and nucleons. In this proceeding we only mention the results of spin-independent interaction. For the spin-dependent interaction discussion, we encourage readers to our published papers $[5,6] . C_{s}$ is the rate at which DM are captured due to their scattering with DM that have already been trapped in the Sun [9],

$$
C_{s}=\sqrt{\frac{3}{2}} n_{\chi} \sigma_{\chi \chi} v_{\mathrm{esc}}\left(R_{\odot}\right) \frac{v_{\mathrm{esc}}\left(R_{\odot}\right)}{\bar{v}}\left\langle\widehat{\phi}_{\chi}\right\rangle \frac{\operatorname{erf}(\eta)}{\eta}
$$


where $\left\langle\widehat{\phi}_{\chi}\right\rangle \simeq 5.1$ [10] is a dimensionless average solar potential experienced by the captured DM within the Sun. $C_{e}$ is the the DM evaporation rate due to DM-nuclei interactions,

$$
C_{e} \simeq \frac{8}{\pi^{3}} \sqrt{\frac{2 m_{\chi}}{\pi T_{\chi}(\bar{r})}} \frac{v_{\mathrm{esc}}^{2}(0)}{\bar{r}^{3}} \exp \left(-\frac{m_{\chi} v_{\mathrm{esc}}^{2}(0)}{2 T_{\chi}(\bar{r})}\right) \Sigma_{\text {evap }}
$$

where $v_{\text {esc }}(0)$ is the escape velocity from the core of the Sun, $T_{\chi}$ is the DM temperature in the Sun, and $\bar{r}$ is average DM orbit radius which is the mean DM distance from the solar center. The quantity $\Sigma_{\text {evap }}$ is the sum of the scattering cross sections of all the nuclei within a radius $r_{95 \%}$, where the solar temperature has dropped to $95 \%$ of the DM temperature. $C_{a}$ is the DM annihilation rate [11],

$$
C_{a} \simeq \frac{\langle\sigma v\rangle V_{2}}{V_{1}^{2}}
$$

where

$$
V_{j} \simeq 6.5 \times 10^{28} \mathrm{~cm}^{3}\left(\frac{10 \mathrm{GeV}}{j m_{\chi}}\right)^{3 / 2}
$$

is the DM effective volume inside the Sun and $\langle\sigma v\rangle$ is the relative velocity averaged annihilation cross section. And $C_{s e}$ is the evaporation rate induced by the interaction between DM particles in the Sun,

$$
C_{s e}=\frac{\int_{\odot} \frac{d C_{s e}}{d V} d^{3} r}{\left(\int_{\odot} n_{\chi}(r) d^{3} r\right)^{2}}
$$

where

$$
\frac{d C_{s e}}{d V}=\frac{4}{\sqrt{\pi}} \sqrt{\frac{m_{\chi}}{2 T_{\chi}}} \frac{n_{0}^{2} \sigma_{\chi \chi}}{m_{\chi}} \exp \left[-\frac{2 m_{\chi} \phi(r)}{T_{\chi}}\right] \exp \left[-\frac{E_{\mathrm{esc}}(r)}{T_{\chi}}\right] \tilde{K}\left(m_{\chi}\right)
$$

and

$$
n_{\chi}(r)=n_{0} \exp \left(-\frac{m_{\chi} \phi(r)}{T_{\chi}}\right)
$$

The coefficients $C_{a, c, e, s, s e}$ are taken to be positive and time-independent.

\section{Testability of SIDM at IceCube-PINGU}

A large parameter space that can be reached the steady DM number in the Sun for the relevant $\sigma_{\chi p}$ and $\sigma_{\chi \chi}$ [5]. Here we concentrate on the probe of SIDM. We consider DM annihilation channels, $\chi \chi \rightarrow \tau^{+} \tau^{-}$and $v \bar{v}$, for producing neutrino final states to be detected by IceCubePINGU [12]. The neutrino differential flux of flavor $i, \Phi_{v_{i}}$, from $\chi \chi \rightarrow f \bar{f}$ can be expressed as

$$
\frac{d \Phi_{v_{i}}}{d E_{v_{i}}}=P_{v_{j} \rightarrow v_{i}}\left(E_{v}\right) \frac{\Gamma_{A}}{4 \pi R_{\odot}^{2}} \sum_{f} B_{f}\left(\frac{d N_{v_{j}}}{d E_{v_{j}}}\right)_{f}
$$


where $R_{\odot}$ is the distance between the neutrino source and the detector, $P_{v_{j} \rightarrow v_{i}}\left(E_{v}\right)$ is the neutrino oscillation probability during the propagation, $B_{f}$ is the branching ratio corresponding to the channel $\chi \chi \rightarrow f \bar{f}, d N_{v} / d E_{v}$ is the neutrino spectrum at the source, and $\Gamma_{A}$ is the DM annihilation rate in the Sun. The neutrino event rate in the detector is given by

$$
N_{v}=\int_{E_{\mathrm{th}}}^{m_{\chi}} \frac{d \Phi_{v}}{d E_{v}} A_{v}\left(E_{v}\right) d E_{v} d \Omega
$$

where $E_{\mathrm{th}}$ is the detector threshold energy, $d \Phi_{v} / d E_{v}$ is the neutrino flux from DM annihilation, $A_{v}$ is the detector effective area, and $\Omega$ is the solid angle. We study both muon track events and cascade events induced by neutrinos. The atmospheric background event rate can also be calculated by Eq. (3.2) with $d \Phi_{v} / d E_{v}$ replaced by the atmospheric neutrino flux. The angular resolution for IceCube-PINGU detector at $E_{v}=5 \mathrm{GeV}$ is roughly $10^{\circ}$ [12]. Hence we consider neutrino events arriving from the solid angle range $\Delta \Omega=2 \pi(1-\cos \psi)$ surrounding the Sun with $\psi=10^{\circ}$. We present the IceCube-PINGU sensitivity to $\sigma_{\chi \chi}$ in the DM mass region $3 \mathrm{GeV}<m_{\chi}<20 \mathrm{GeV}$ for SI cases in Fig. 1. The sensitivities to $\sigma_{\chi \chi}$ are taken to be $2 \sigma$ significance for 5 years of data taking. The shadow areas in the figures represent those parameter spaces disfavored by the Bullet Cluster and halo shape analyses. Below the black solid line, the DM self-interaction is too weak to resolve the core/cusp problem of the structure formation. Two benchmark values of thermal average cross section, $\langle\sigma v\rangle=3 \times 10^{-26} \mathrm{~cm}^{3} \mathrm{~s}^{-1}$ and $\langle\sigma v\rangle=3 \times 10^{-27} \mathrm{~cm}^{3} \mathrm{~s}^{-1}$ are used for our studies. One can see that a large parameter space is covered by the IceCube-PINGU sensitivities.

\section{Direct and indirect complementary detection of SIDM - A case of dark U(1) model}

We then apply a simple hidden $U(1)$ gauge symmetry and $\phi_{\mu}$ is the corresponding gauge boson that mediate dark force among DM. The relevant effective Lagragian can be written as

$$
L_{\text {mixing }, U(1)}=\frac{\varepsilon_{\gamma}}{2} \phi_{\mu v} F^{\mu v}+\varepsilon_{Z} m_{Z}^{2} \phi_{\mu} Z^{\mu}
$$

where the parameters $\varepsilon_{\gamma}$ and $\varepsilon_{Z}$ are originated from kinetic and mass mixings between $\phi^{\mu}$ and photon and $Z$ boson. The SI cross section between DM and any nucleus with mass number $A$ and proton number $Z$ is then given by

$$
\sigma_{\chi A} \approx \frac{16 \pi \alpha_{\chi} \alpha_{\mathrm{em}}}{m_{\phi}^{4}}\left[\varepsilon_{p} Z+\varepsilon_{n}(A-Z)\right]^{2} \mu_{\chi A}^{2},
$$

with $\alpha_{\mathrm{em}}$ the fine structure constant, $\mu_{\chi A} \equiv m_{\chi} m_{A} /\left(m_{\chi}+m_{A}\right)$ the reduced mass for DM-nucleus system and $\alpha_{\chi} \equiv e_{D}^{2} /(4 \pi)$ is the fine structure constant in the hidden $U(1)$ sector. The effective $\phi_{\mu}$-nucleon couplings, $\varepsilon_{p}$ and $\varepsilon_{n}$, can be expressed in terms of $\varepsilon_{\gamma}$ and $\varepsilon_{Z}$ such that [13]

$$
\begin{aligned}
& \varepsilon_{p}=\varepsilon_{\gamma}+\frac{\varepsilon_{Z}}{4 s_{W} c_{W}}\left(1-4 s_{W}^{2}\right) \approx \varepsilon_{\gamma}+0.05 \varepsilon_{Z}, \\
& \varepsilon_{n}=-\frac{\varepsilon_{Z}}{4 s_{W} c_{W}} \approx-0.6 \varepsilon_{Z} .
\end{aligned}
$$

Therefore, isospin violation is generally introduced in the hidden $U(1)$ model since the ratio $\varepsilon_{n} / \varepsilon_{p}$ can be any number [14]. Let us define $\varepsilon_{n} / \varepsilon_{p} \equiv \eta$, with $\eta=1$ for isospin symmetric case and 

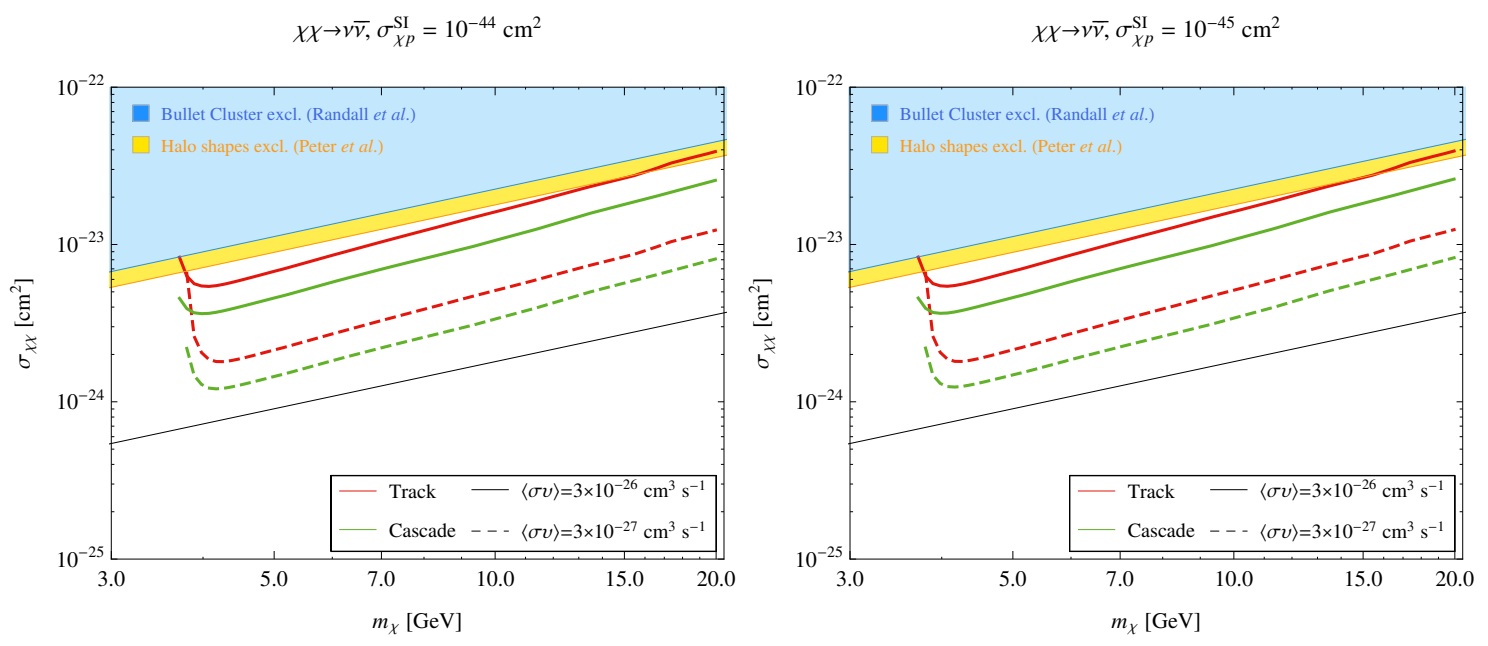

$\chi \chi \rightarrow \tau^{+} \tau^{-}, \sigma_{\chi p}^{\mathrm{SI}}=10^{-44} \mathrm{~cm}^{2}$
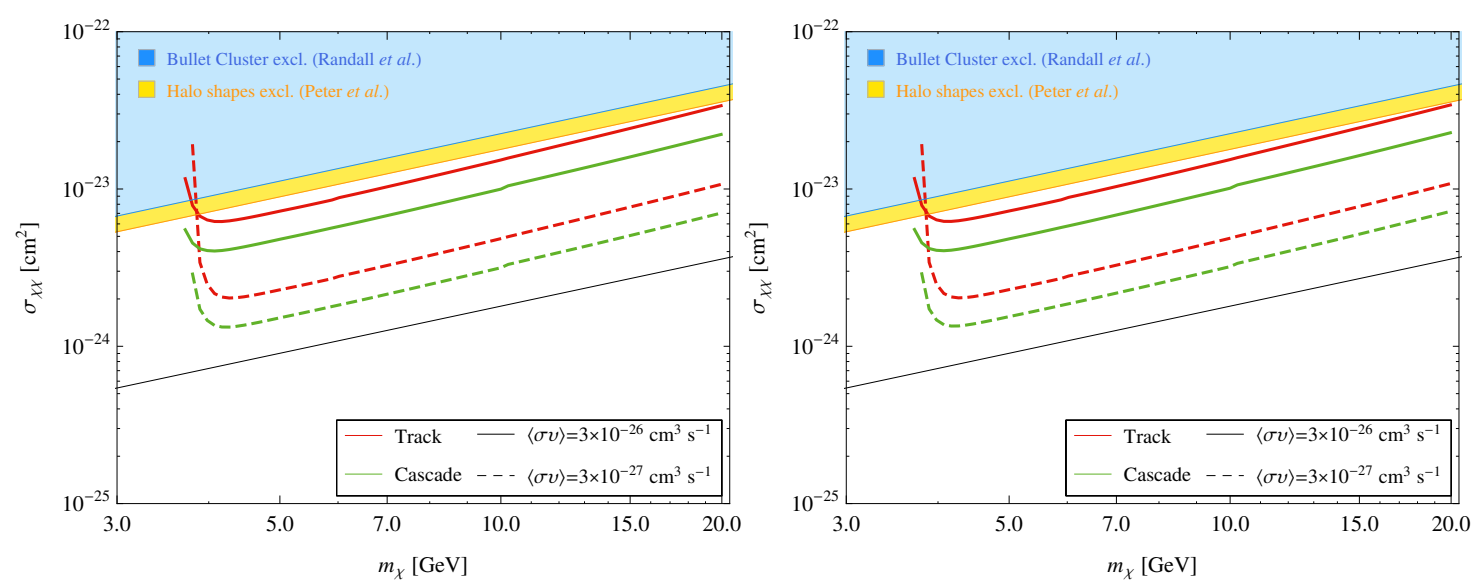

Figure 1: The IceCube-PINGU sensitivities to DM self-interaction cross section $\sigma_{\chi \chi}$ as a function of $m_{\chi}$. The DM-nucleus interaction inside the Sun is assumed to be dominated by SI interaction.

$\eta=-0.7$ for the maximized isospin violation with respect to Xe element. And $\varepsilon_{\gamma}=10^{-9}$ stands for the DM-nuclei cross section that is within the next generation direct detection region while $\varepsilon_{\gamma}=10^{-10}$ represents the case of DM-nuclei cross section which is suppressed. The evolution behaviors of $N_{\chi}$ with different $m_{\chi}$ and $\eta$ for $m_{\phi}=30 \mathrm{MeV}$ are shown in Fig. 2. One can see that the total trapped DM numbers are slightly less for the suppressed $\sigma_{\chi p}$ case but still provide enough indirect signals. Although one should notice that the accumulation time is also longer for the small $\sigma_{\chi p}$ scenario. Neutrino signals arise from the decays of $\phi$ which is produced by DM annihilation, $\chi \bar{\chi} \rightarrow \phi \phi \rightarrow 4 v$. For the hidden $U(1)$ gauge model we consider in this paper, the branching ratio for $\phi \rightarrow v \bar{v} \approx 75 \%, 39 \%, 48 \%$ and $67 \%$ for $\eta=1,-0.3,-0.5$ and -0.7 , respectively. The lifetime of $\phi$ is constrained by Big Bang Nucleosynthesis (BBN), $\tau_{\phi} \lesssim \mathscr{O}(1) \mathrm{s}$, in order to produce extra degrees of freedom. Considering a $30 \mathrm{MeV} \phi$ with $5 \mathrm{GeV}$ energy (corresponding to $m_{\chi}=10 \mathrm{GeV}$ ), the decay time of $\phi$ would be less than $170 \mathrm{~s}$. One can easily estimated that, for a $\phi$ with $170 \mathrm{~s}$ of decay time, it travels for roughly $5 \times 10^{7} \mathrm{~km}$ before its decay into electron-position or neutrino pair. The decay point is already outside the Sun but not yet reaching to the Earth. Given the distance 

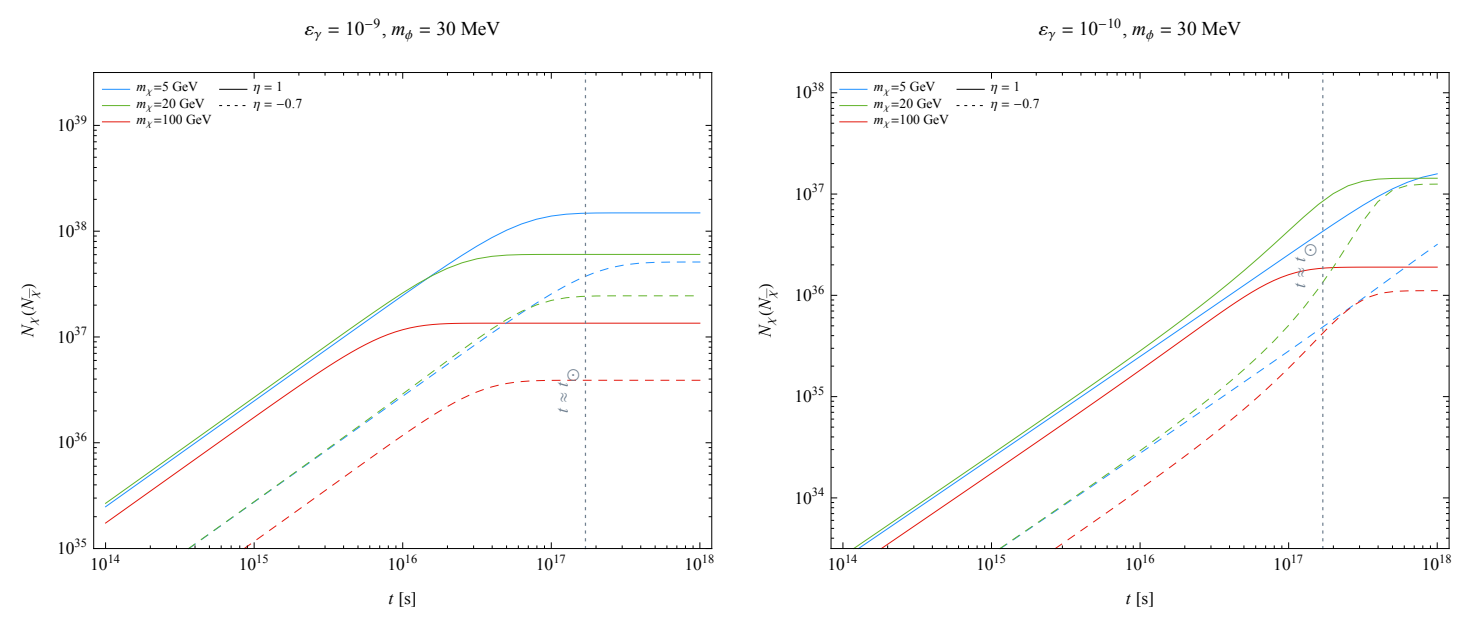

Figure 2: The $N_{\chi}\left(N_{\bar{\chi}}\right)$ for $\varepsilon_{\gamma}=10^{-9}$ and $10^{-10}$ for $m_{\phi}=30 \mathrm{MeV}$. Solid lines are for isospin symmetric case and dashed lines are for isospin violated case. Blue, green and red curves are for $m_{\chi}=$ $5 \mathrm{GeV}, 20 \mathrm{GeV}$ and $100 \mathrm{GeV}$, respectively. The solar age $\left(t_{\odot}\right)$ is indicated by the gray dashed line.

between the Sun and the Earth at $1.5 \times 10^{8} \mathrm{~km}$, those $\phi$ with $30 \mathrm{MeV}$ of mass and moving toward the Earth shall decay between Sun and Earth provided $E_{\phi}$ is less than $150 \mathrm{GeV}$. Hence the neutrino flux will be observed by the terrestrial detector. Using similar calculation for the expective neutrino signals and atmospheric background estimation we present the IceCube-PINGU sensitivities to $m_{\phi}$ with different values of $\eta$ in colored solid lines in Fig. 3. Regions above these lines are beyond the reach of the detector within a $2 \sigma$ detection significance in 5 years. The left panel is for cascade events and the right one is for track events. Furthermore, each sensitivity curve terminates at the evaporation mass scale below which the DM signature from the Sun is suppressed. BBN excludes $m_{\phi}<20 \mathrm{MeV}$ for $\varepsilon_{\gamma}=10^{-10}$, while it exludes $m_{\phi}<0.3 \mathrm{MeV}$ for $\varepsilon_{\gamma}=10^{-9}$. For simplicity, we only present results for $\varepsilon_{\gamma}=10^{-9}$ here. The orange band is SIDM allowed region. The colored shaded regions are exluded by LUX for differnet $\eta$ values. The gray dashed lines indicate the relation between $\alpha_{\chi}$ and $m_{\chi}$ required by the thermal relic density. density. It is of interest to compare results with different $\eta$ values. It is seen that the sensitivity curve with $\eta=1$ differs significantly from those with other $\eta$ values. However, sensitivity curevs with $-0.3 \leq \eta \leq-0.7$ do not differ much from one another. Compare left and right panels of Fig. 3, one can see that cascade events can probe a larger part of $m_{\chi}-m_{\phi}$ parameter space. We notice that the IceCubePINGU sensitivity with LUX constraint is significantly weaken by isospin violation. As one can see that the LUX excluded region shrinks as $\eta$ decreases from 1 to -0.7 . On ther other hand, the indirect DM signature from the Sun is less affected by $\eta$. Hence the complementarity of two searches becomes more apparent when $\sigma_{\chi p}$ is subject to more severe isospin violation suppression.

\section{Summary}

In summary, we have studied the time evolution of DM number trapped inside the Sun with SIDM considered. We found that the inclusion of SIDM can increase the number of trapped DM and shorten the accumulation time. Most of the SIDM parameter space which is allowed by the 

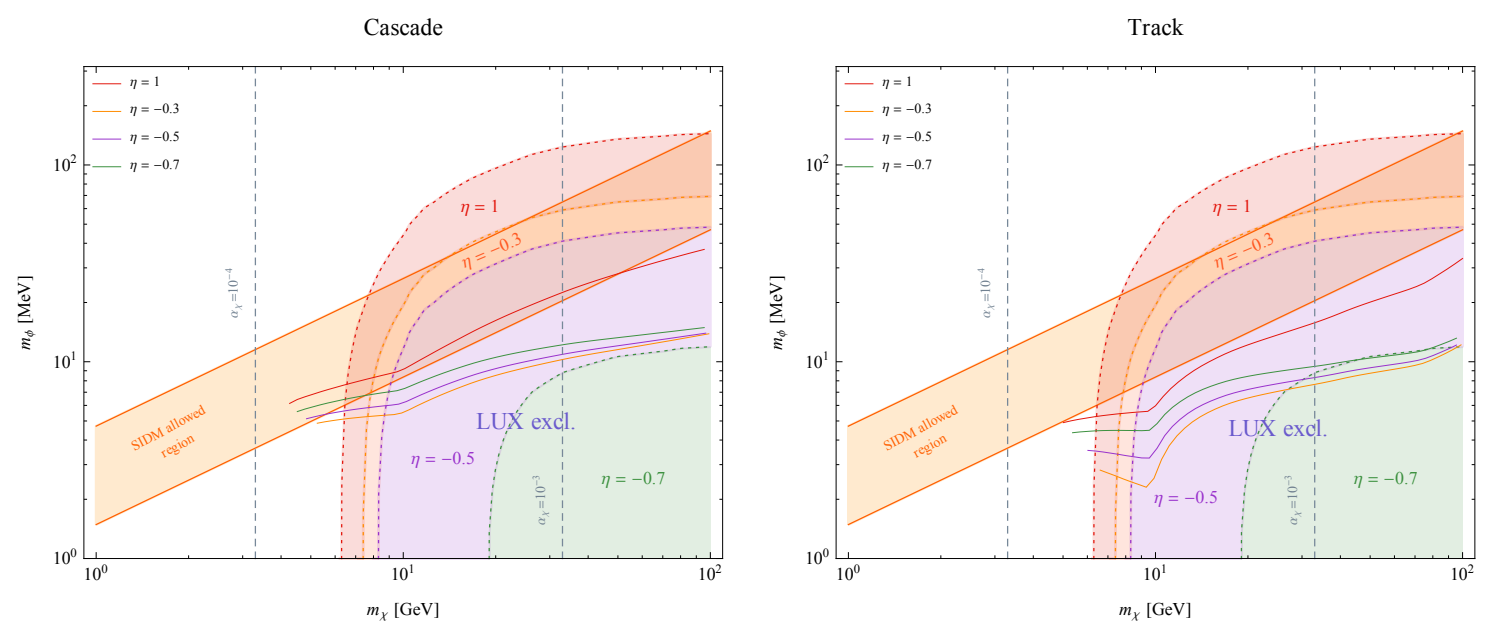

Figure 3: The IceCube-PINGU sensitivities to $m_{\phi}$ for different values of $\eta$. The left panel is for track events and the right panel is for cascade events. The sensitivity curves and LUX excluded regions correspond to $\varepsilon_{\gamma}=10^{-9}$. The results corresponding to $\varepsilon_{\gamma}=10^{-10}$ are not shown since BBN constraint rules out most of the $m_{\phi}$ parameter space plotted here.

astrophysical observations can be probed by IceCube-PINGU if DM annihilate into neutrino final states via the channels $\chi \chi \rightarrow v \bar{v}\left(\tau^{+} \tau^{-}\right)$. We further demonstrate that DM accumulation in the Sun is dominated by SIDM when $\sigma_{\chi p}$ is highly suppressed. In this case, the total number of captured DM is not significantly reduced such that the strength of indirect signal is insensitive to $\sigma_{\chi p}$.

\section{References}

[1] D. N. Spergel and P. J. Steinhardt, Observational evidence for selfinteracting cold dark matter, Phys. Rev. Lett.84 (2000) 3760 [astro-ph/9909386].

[2] W. J. G. de Blok, The Core-Cusp Problem, Adv. Astro.2010, 789293 (2010) [arXiv: 0910 . 3538].

[3] M. G. Walker, [arXiv:1205.0311].

[4] M. Boylan-Kolchin, J. S. Bullock and M. Kaplinghat, Too big to fail? The puzzling darkness of massive Milky Way subhaloes, Mon. Not. Roy. Astron. Soc.415, L40 (2011) [arXiv:1103. 0007].

[5] C. S. Chen, F. F. Lee, G. L. Lin and Y. H. Lin, Probing Dark Matter Self-Interaction in the Sun with IceCube-PINGU, JCAP1410 (2014) 10, 049 [arXiv: 1408.5471 [hep-ph]].

[6] C. S. Chen, G. L. Lin and Y. H. Lin, Complementary Test of the Dark Matter Self-Interaction by Direct and Indirect Detections, arXiv:1505.03781 [hep-ph].

[7] G. Jungman, M. Kamionkowski and K. Griest, Supersymmetric dark matter, Phys. Rept. 267, 195 (1996) [hep-ph/9506380].

[8] G. Bertone, D. Hooper and J. Silk, Particle dark matter: Evidence, candidates and constraints, Phys. Rept. 405, 279 (2005) [hep-ph/ 0404175$].$

[9] A. R. Zentner, High-Energy Neutrinos From Dark Matter Particle Self-Capture Within the Sun, Phys. Rev. D80 (2009) 063501 [arXiv: 0907.3448 [astro-ph.HE]]. 
[10] A. Gould, Cosmological density of WIMPs from solar and terrestrial annihilations, Astrophys. J.388 (1992) 338.

[11] K. Griest and D. Seckel, Cosmic Asymmetry, Neutrinos and the Sun, Nucl. Phys. B283, 681 (1987) [Erratum-ibid. B 296, 1034 (1988)].

[12] M. G. Aartsen et al. [IceCube-PINGU Collaboration], arXiv:1401.2046 [physics.ins-det] (2014).

[13] M. Kaplinghat, S. Tulin and H. B. Yu, Direct Detection Portals for Self-interacting Dark Matter, Phys. Rev. D89 (2014) 3, 035009 [arXiv: 1310.7945 [hep-ph]].

[14] M. T. Frandsen, F. Kahlhoefer, S. Sarkar and K. Schmidt-Hoberg, Direct detection of dark matter in models with a light Z', JHEP1109 (2011) 128 [arXiv: 1107.2118 [hep-ph]]. 